\title{
Remaining spine deformity after revision surgery for pelvic reconstruction and spinopelvic fixation: illustrative case
}

\author{
Takayuki Ito, MD, Shunsuke Fujibayashi, MD, PhD, Bungo Otsuki, MD, PhD, Shimei Tanida, MD, PhD, Takeshi Okamoto, MD, PhD, and \\ Shuichi Matsuda MD, PhD
}

Department of Orthopedic Surgery, Graduate School of Medicine, Kyoto University, Kyoto, Japan

BACKGROUND Pelvic deformity after resection of malignant pelvic tumors causes scoliosis. Although the central sacral vertical line (CSVL) is often used to evaluate the coronal alignment and determine the treatment strategy for scoliosis, it is not clear whether the CSVL is a suitable coronal reference axis in cases with pelvic deformity. This report proposes a new coronal reference axis for use in cases with pelvic deformity and discusses the pathologies of spinal deformity remaining after revision surgery.

OBSERVATIONS A 14-year-old boy who had undergone internal hemipelvectomy and pelvic ring reconstruction 2 years prior was referred to our hospital with severe back pain. His physical and radiographic examinations revealed severe scoliosis with pelvic deformity. The authors planned a surgical strategy based on the CSVL and performed pelvic ring reconstruction using free vascularized fibula graft and spinopelvic fixation from L5 to the pelvis. After the procedure, although the patient's back pain was relieved, his scoliosis persisted. At the latest follow-up, his spinal deformity correction was acceptable with corset bracing. Therefore, the authors did not perform additional surgeries.

LESSONS The CSVL may not be appropriate for evaluating coronal alignment in cases with pelvic deformity. Accurate preoperative planning is required to correct spinal deformities with pelvic deformity.

https://thejns.org/doi/abs/10.3171/CASE21209

KEYWORDS scoliosis; spine deformity; pelvic deformity; coronal alignment; pelvic malignant bone tumor

Malignant pelvic tumors remain a major challenge for orthopedic surgeons. External hemipelvectomy, a hindquarter amputation, is traditionally performed for tumor resection. With improvements in multidrug chemotherapy, limb-sparing tumor resection has also been performed. ${ }^{1}$ In Enneking grade I or I + IV tumors, reconstruction of the pelvic ring is critical for maintaining the pelvis and spinal column stability. Several reconstruction procedures have been reported, including vascularized or nonvascularized fibula autografts, ${ }^{2-5}$ vascularized iliac grafts, ${ }^{6}$ saddle prostheses, and three-dimensional (3D)-printed custom-made prostheses. ${ }^{7}$ However, each of these techniques is associated with unique complications, and there remains no standard treatment. Additionally, the long-term oncological and functional outcomes remain unknown. Furthermore, no studies have reported on revision surgery for pelvic ring reconstruction to treat secondary scoliosis caused by pelvic deformity after internal hemipelvectomy.

Several authors have reported scoliosis after hemipelvectomy. ${ }^{2,8}$ Papanastassiou et al. ${ }^{8}$ reported the use of spinopelvic fixation to treat scoliosis with pelvic deformity after hemipelvectomy. They evaluated coronal alignment using the central sacral vertical line (CSVL). However, they did not refer to the pelvic deformity and did not report how to determine the upper instrumented vertebrae. Although the CSVL is often used to evaluate the coronal alignment and determine the treatment strategy, it is not clear whether the CSVL is a suitable coronal reference axis in cases with pelvic deformity.

This report proposes a new coronal reference axis for use in cases with pelvic deformity and discusses the pathologies of spinal deformity remaining after revision surgery.

ABBREVIATIONS 3D $=$ three-dimensional; $\mathrm{C7PL}=\mathrm{C7}$ plumb line; $\mathrm{CSVL}=$ central sacral vertical line; $\mathrm{CT}=$ computed tomography; $\mathrm{PBFH}=$ perpendicular line of the bilateral femoral heads; $S V=$ stable vertebra.

INCLUDE WHEN CITING Published July 12, 2021; DOI: 10.3171/CASE21209.

SUBMITTED April 6, 2021. ACCEPTED May 5, 2021.

(C) 2021 The authors, CC BY-NC-ND 4.0 (http://creativecommons.org/licenses/by-nc-nd/4.0/). 

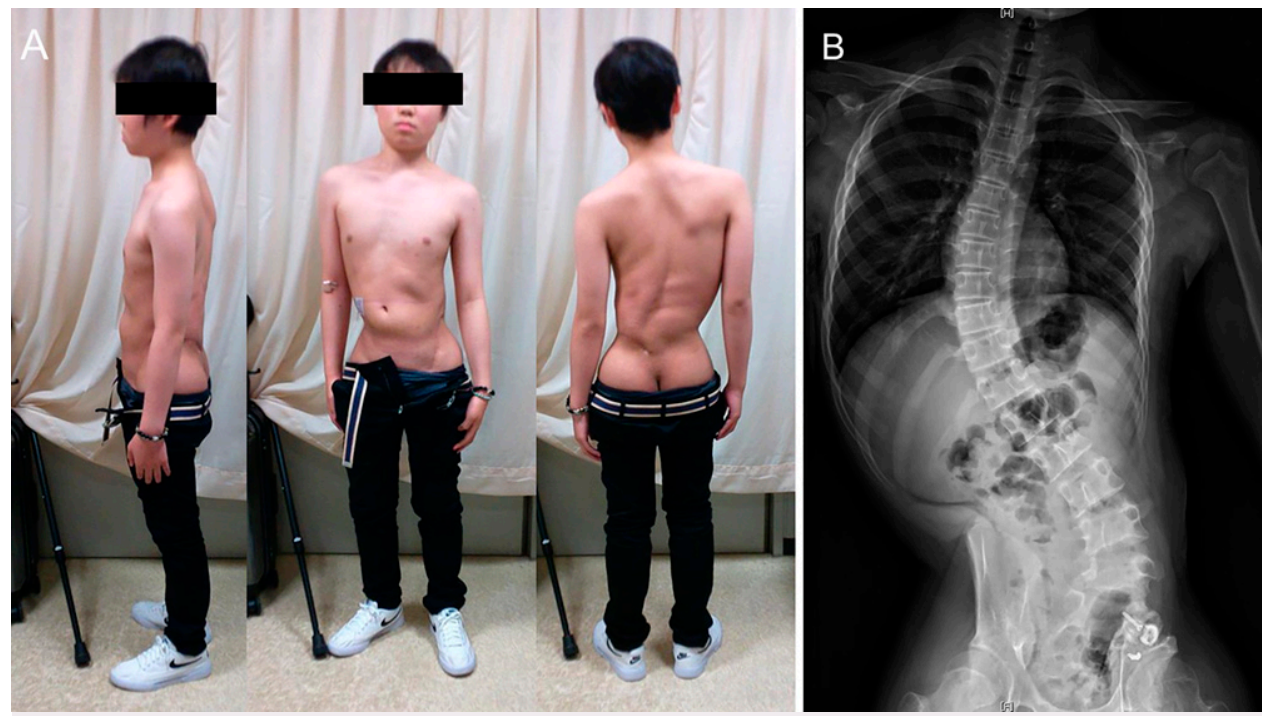

FIG. 1. A: Preoperative photographs showing coronal malalignment with a left lumbar curve and right rib prominence. B: Preoperative radiograph showing scoliosis and pelvic deformity after graft nonunion.

\section{Illustrative Case}

\section{Patient History and Examination}

A 14-year-old boy was referred to our hospital with a complaint of severe back pain. When he was 12 years old, he had been diagnosed with Ewing sarcoma of the left ilium (Enneking classification P1). In combination with neoadjuvant and adjuvant chemotherapy, the patient underwent tumor resection ( $\mathrm{P} 1$ resection) and reconstruction of the pelvic ring with free nonvascularized fibular autograft. However, he developed graft failure and secondary scoliosis.

The patient was barely able to walk with a Lofstrand crutch and required significant use of opioids, nonsteroidal antiinflammatory drugs, and psychotropic drugs for his severe back pain. Visual inspection (Fig. 1A) showed a left lumbar curve and right rib prominence.

Radiographs revealed nonunion of the autograft, pelvic ring deformity, and spinal scoliosis (Fig. 1B). In standing posteroanterior radiographs, the thoracic and lumbar compensatory curves measured $56^{\circ}$ (T4 to L2) and $45^{\circ}$ (L2 to L5), respectively. The distance between the C7 plumb line (C7PL) and the CSVL was $55 \mathrm{~mm}$, which we considered a coronal imbalance. The other spinopelvic parameters were as follows: sagittal vertical axis (C7-S1) $7.2 \mathrm{~mm}$, thoracic kyphosis $4.0^{\circ}$, lumbar lordosis $31^{\circ}$, sacral slope $21^{\circ}$, pelvic tilt $48^{\circ}$, and pelvic incidence $69^{\circ}$ (Fig. 2A and B). A right-bending film showed that the
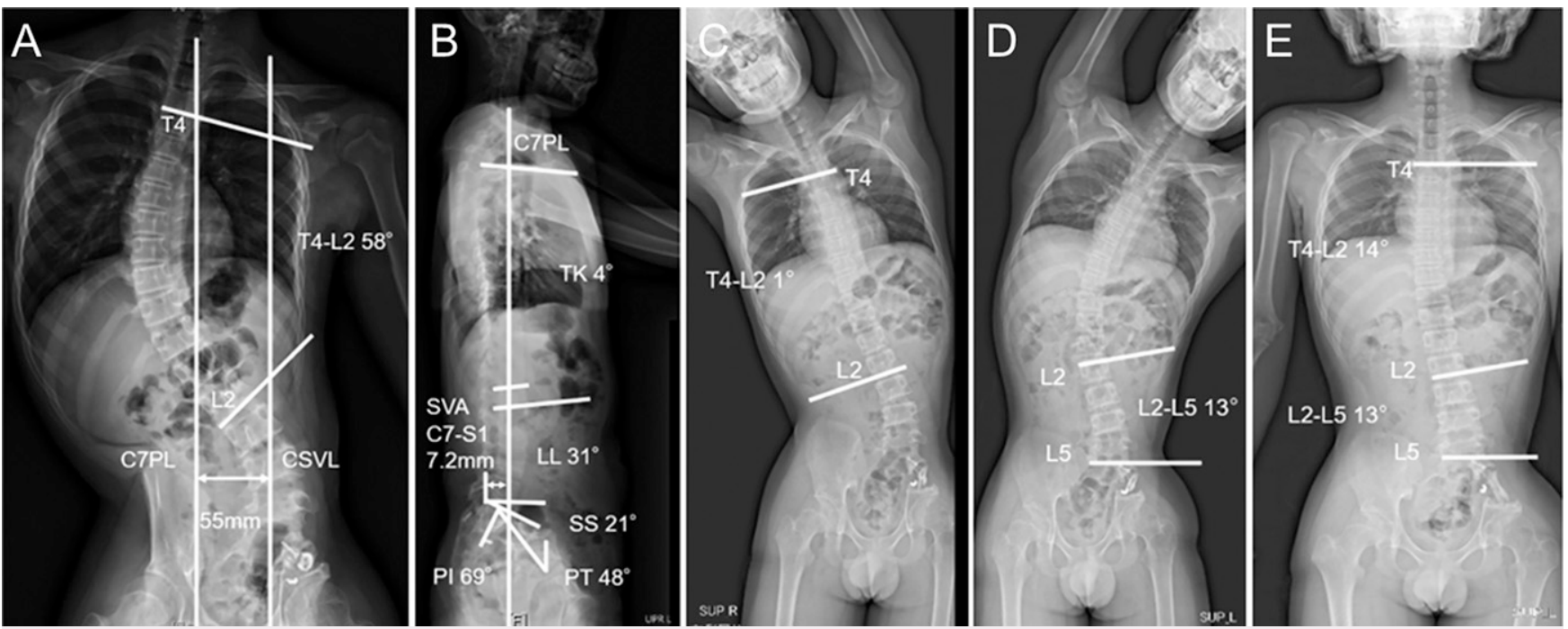

FIG. 2. A and B: Preoperative anteroposterior (A) and lateral (B) standing whole-spine radiographs showing $56^{\circ}$ thoracic compensatory curve from $\mathrm{T} 4$ to L2 and coronal malalignment. The other spinopelvic parameters are C7PL-CSVL $55 \mathrm{~mm}$, C7-sagittal vertical axis (SVA) $+7.2 \mathrm{~mm}$, thoracic kyphosis (TK) $4^{\circ}$, lumbar lordosis (LL) $31^{\circ}$, sacral slope (SS) $21^{\circ}$, pelvic tilt (PT) $48^{\circ}$, pelvic incidence (PI) $69^{\circ}$. C: Preoperative anteroposterior right-bending whole-spine radiograph showing correction of the thoracic compensatory curve to $1^{\circ}$. D: Preoperative anteroposterior left-bending whole-spine radiographs showing correction of the lumber compensatory to $13^{\circ}$. E: Preoperative anteroposterior traction whole-spine radiograph showing correction of the thoracic and lumbar curves to $14^{\circ}$ and $13^{\circ}$, respectively. 
thoracic compensatory curve was so flexible that it was corrected to $1^{\circ}$. His left-bending film showed a less flexible lumbar compensatory curve that was corrected to $13^{\circ}$. Traction radiographs demonstrated that both curves were corrected (Fig. 2C-E).

The patient was diagnosed with nonstructural scoliosis related to pelvic deformity after resection of a malignant pelvic bone tumor and graft nonunion. It was difficult to understand the relationship between the pelvic structures, including the internal iliac artery/vein, urinary duct, and failure graft. A model of the patient's pelvis was created on a 3D printer for preoperative planning. We performed revision surgery for pelvic ring reconstruction with a vascularized fibula graft and spinopelvic fixation from $L 5$ to the pelvis.

\section{Operation}

The patient was placed in a floppy lateral position on an operating frame connected to an intraoperative computed tomography (CT) scanner using a navigation system. The failed graft was exposed and removed through the same skin incision as in the first operation. The preoperative posteroanterior whole-spine radiograph showed that the stable vertebra (SV), an important marker for determining fusion levels, was L5 according to the CSVL. The upper instrumented vertebra was determined to be at L5. Because we believed that screw insertion into the remnant of the left iliac bone would not be sufficient to rigidly fix the transferred bone, we instead inserted the screws into the left ischium and pubis using intraoperative CT navigation. After the rod connection was performed, the $L 5$ vertebral body appeared horizontal on an intraoperative radiograph in the supine position without the application of special correction techniques.

A free vascularized fibular graft was harvested from the contralateral side and placed between the remnant of the left iliac bone and the sacrum by a microsurgery team. The vascular pedicle was anastomosed with a branch of the inferior gluteal artery and vein. The transferred bone graft was then fixed using cannulated screws.

\section{Postoperative Course}

The patient's back pain was relieved, and graft union was achieved (Fig. 3A and B). However, his scoliosis persisted (Fig. 3C).
The thoracic and lumbar curves were $58^{\circ}$ and $30^{\circ}$, respectively. We used brace treatment to treat the scoliosis. At the latest followup ( 3 years after revision surgery), the thoracic curve was $35^{\circ}$ without the brace and $25^{\circ}$ with the brace (Fig. 3D and E). The patient was unable to continue wearing the brace but did not show any progress of deformity or exacerbation of pain at his 3-year followup. Therefore, no surgical correction was performed, but if the deformity progresses in the future, surgical treatment should be considered. Careful consideration is required because of the high risk of disability in activities of daily living due to long-segment fixation and occurrence of adjacent segment disorders due to pelvic deformity.

\section{Discussion}

\section{Observations}

This is the first case report of revision surgery for pelvic ring reconstruction for secondary scoliosis after the resection of a malignant pelvic tumor. Although some authors reported that the functional scores and nononcologic complications after reconstruction were not superior to those without reconstruction, ${ }^{9}$ the failure of reconstruction in our case led to pelvic deformity and secondary scoliosis. We believe that pelvic ring reconstruction is mandatory after tumor resection. The reconstruction procedures include vascularized iliac autografts, strut allografts, autoclaved autogenous bone grafts, vascularized or nonvascularized fibula grafts, and patient-specific prostheses. ${ }^{2-7,10}$ We used a vascularized fibular graft to achieve complete bone union.

Akiyama et al. reported that pelvic ring reconstruction without spinal instrumentation could achieve graft union in all patients. ${ }^{5}$ However, in our case, pelvic ring reconstruction without spinal instrumentation at the first surgery in another hospital caused graft failure and pelvic deformity. We think that accurate anatomical reconstruction of the pelvic ring and rigid fixation with spinal instrumentation after resection of malignant pelvic tumors are very important in order not to cause pelvic deformity.

Ogura et al. reported that the use of a vascularized fibula graft and spinal instrumentation showed good functional outcomes and a high rate of bone union. However, screws are inserted into the
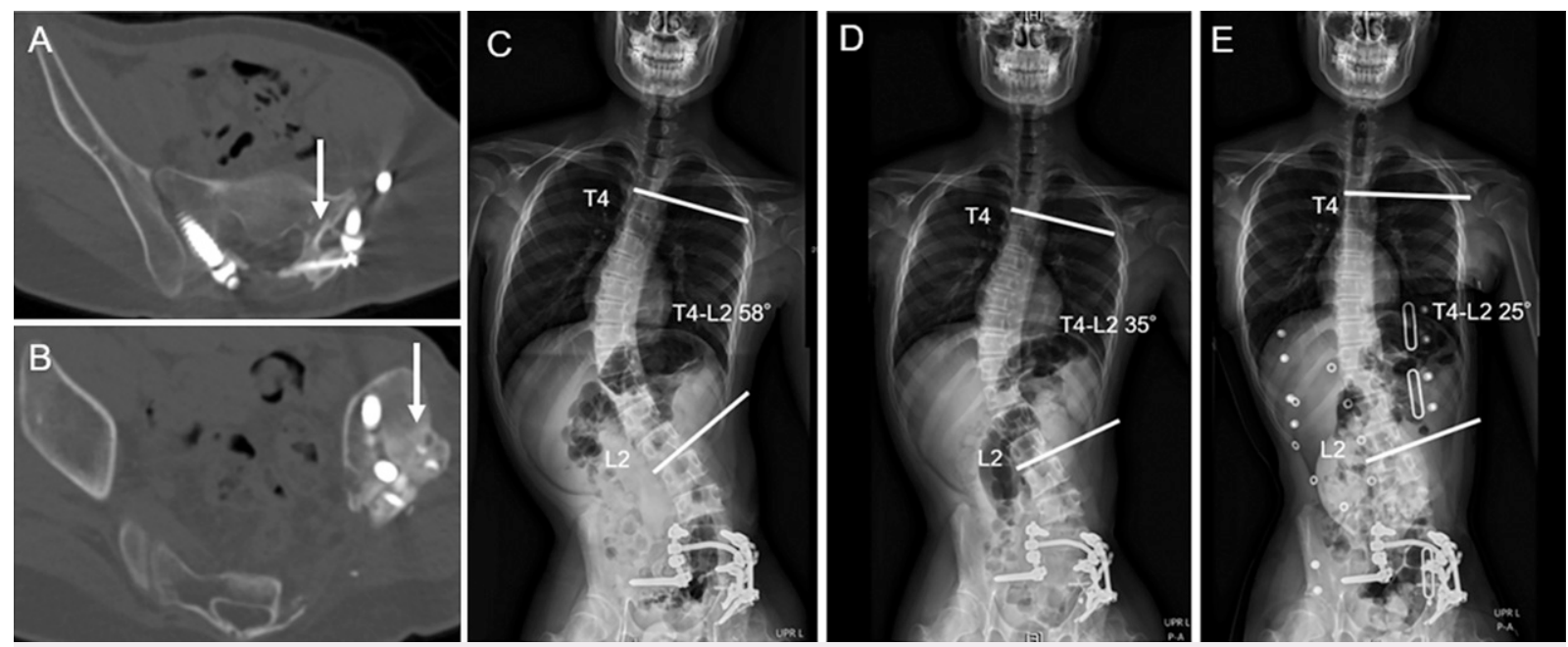

FIG. 3. A and B: Postoperative CT axial views showing complete bone union (arrows). C: Immediate postoperative anteroposterior standing whole-spine radiograph showing a thoracic curve of $58^{\circ}$. D: Anteroposterior standing whole-spine radiograph at the latest follow-up showing a thoracic curve of $35^{\circ}$. E: Anteroposterior standing whole-spine radiograph at the latest follow-up with corset bracing showing a thoracic curve of $25^{\circ}$. 

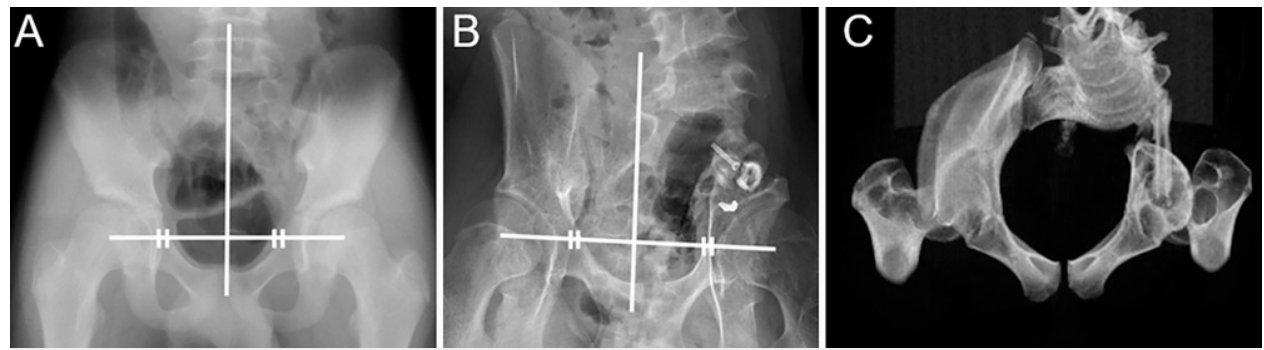

FIG. 4. A: Anteroposterior radiograph of the pelvis before tumor resection showing the sacrum in a normal position. $\mathbf{B}$ and $\mathbf{C}$ : Anteroposterior radiograph $(\mathbf{B})$ and $3 \mathrm{D}$ CT $(\mathbf{C})$ of the pelvis before revision surgery with a sacrum shift to the left side.

residual iliac bone. ${ }^{2}$ We believe that our method allows the insertion of longer screws and achieves rigid fixation.

Although pelvic stability was achieved and the patient's back pain was relieved, the patient's nonstructural scoliosis persisted. The following three points may explain this outcome. First, the pelvic deformity remained after the revision surgery. Second, treatment of the scoliosis required correction of the upper spine levels. Third, determining the SV and upper instrumented vertebrae on the basis of the CSVL may not be correct.

In retrospect, a radiograph showed that the sacrum had gradually shifted to the left side after the first operation (Fig. 4). Although we achieved stabilization of the pelvis and healing of the bone graft, this was not sufficient for the correction of the pelvic deformity. Because the pelvic deformity was already structural, it contributed to the persisting scoliosis.

In general, the psoas major, iliacus, and paravertebral muscles support the spinal column against gravity. However, these muscles were resected with the tumor in the present case; thus, it was difficult to stabilize the spinal column. Therefore, fixation of the spinal column might be necessary to correct scoliosis.

However, it is not clear how to determine fusion levels. The CSVL is often used to evaluate coronal alignment and determine the SV. Because the distance between the C7PL and CSVL was 55 $\mathrm{mm}$ in the present case, we considered the preoperative coronal alignment to be imbalanced. In addition, the SV was L5 according to the CSVL. Considering the pelvic deformity with shifting of the sacrum, the CSVL was not suitable for the evaluation of coronal alignment and SV determination. Thus, cases of scoliosis with pelvic deformity require a new reference axis to evaluate coronal alignment. The postoperative picture of the standing posture showed that the patient's head was positioned over the center of the pelvis, and he did not seem to have a coronal imbalance (Fig. $5 \mathrm{~A})$. Moreover, the postoperative standing radiograph showed that the C7PL matched the perpendicular line of the bilateral femoral heads (PBFH) (Fig. 5B). Therefore, according to PBFH, the coronal alignment was good. If the correction of the spinal deformity had not been acceptable with brace treatment, additional surgery would have been required. The preoperative standing and traction radiographs showed a coronal imbalance, and the SV was L2 on the basis of PBFH (Fig. 5C and D). Adding fixation from the pelvis to L2 might be necessary in this case. Thus, the PBFH was a more appropriate reference axis than the CSVL to evaluate coronal alignment in a case with pelvic deformity. Further follow-up is necessary to determine whether spinal deformities will develop.

\section{Lessons}

In summary, we report a case of revision surgery for scoliosis with pelvic deformity after malignant Ewing sarcoma of the ilium. Reconstruction of the pelvic ring and rigid fixation with spinal instrumentation are important after resection of malignant pelvic tumors
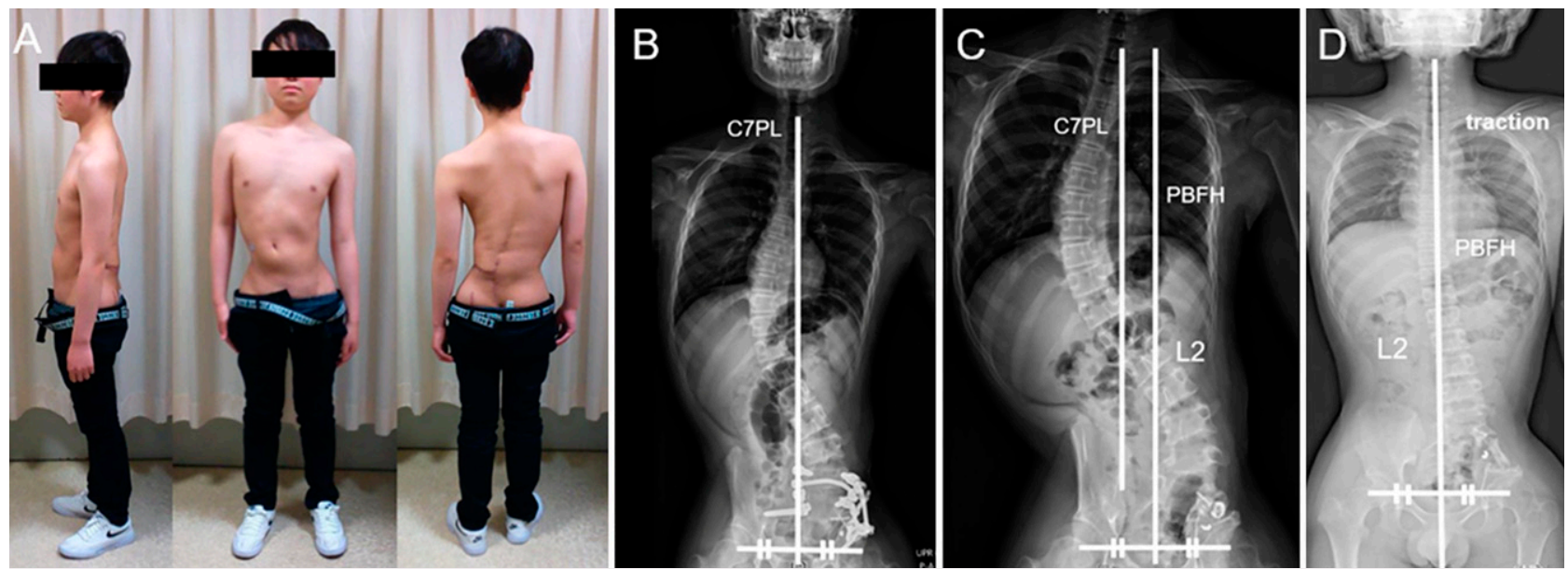

FIG. 5. A: Postoperative photographs showing the location of the patient's head over the center of the pelvis. B: Postoperative standing wholespine radiograph showing that the C7PL matches the PBFH. C and D: Preoperative anteroposterior standing (C) and traction (D) whole-spine radiographs showing $\mathrm{L} 2$ as the SV based on the $\mathrm{PBFH}$. 
in order not to cause structural pelvic deformity and subsequent scoliosis. If spinal deformities with structural pelvic deformity happen, accurate preoperative planning is required. PBFH might be more useful than the CSVL in evaluating the coronal alignment in cases with pelvic deformity.

\section{References}

1. Wilson RJ, Freeman TH Jr, Halpern JL, et al. Surgical outcomes after limb-sparing resection and reconstruction for pelvic sarcoma a systematic review. JBJS Rev. 2018;6(4):e10.

2. Ogura K, Sakuraba M, Miyamoto $S$, et al. Pelvic ring reconstruction with a double-barreled free vascularized fibula graft after resection of malignant pelvic bone tumor. Arch Orthop Trauma Surg. 2015;135(5):619-625.

3. Sakuraba $\mathrm{M}$, Kimata $\mathrm{Y}$, lida $\mathrm{H}$, et al. Pelvic ring reconstruction with the double-barreled vascularized fibular free flap. Plast Reconstr Surg. 2005;116(5):1340-1345.

4. Chang DW, Fortin AJ, Oates SD, Lewis VO. Reconstruction of the pelvic ring with vascularized double-strut fibular flap following internal hemipelvectomy. Plast Reconstr Surg. 2008;121(6): 1993-2000.

5. Akiyama T, Clark JC, Miki Y, Choong PF. The non-vascularised fibular graft: a simple and successful method of reconstruction of the pelvic ring after internal hemipelvectomy. J Bone Joint Surg Br. 2010;92(7):999-1005.

6. Sabourin M, Biau D, Babinet A, et al. Surgical management of pelvic primary bone tumors involving the sacroiliac joint. Orthop Traumatol Surg Res. 2009;95(4):284-292.

7. Liang $\mathrm{H}$, Ji T, Zhang Y, et al. Reconstruction with 3D-printed pelvic endoprostheses after resection of a pelvic tumour. Bone Joint $\mathrm{J}$. 2017;99-B(2):267-275.

8. Papanastassiou I, Boland PJ, Boachie-Adjei O, et al. Scoliosis after extended hemipelvectomy. Spine (Phila Pa 1976). 2010;35(23):E1328-E1333.

9. Schwameis E, Dominkus M, Krepler P, et al. Reconstruction of the pelvis after tumor resection in children and adolescents. Clin Orthop Relat Res. 2002;(402):220-235.

10. Harrington KD. The use of hemipelvic allografts or autoclaved grafts for reconstruction after wide resections of malignant tumors of the pelvis. J Bone Joint Surg Am. 1992;74(3):331-341.

\section{Disclosures}

The authors report no conflict of interest concerning the materials or methods used in this study or the findings specified in this paper.

\section{Author Contributions}

Conception and design: Fujibayashi, Tanida, Matsuda. Acquisition of data: Fujibayashi, Tanida. Analysis and interpretation of data: Fujibayashi, Otsuki, Tanida. Drafting the article: Ito, Tanida. Critically revising the article: Otsuki, Tanida, Matsuda. Reviewed submitted version of manuscript: Fujibayashi, Otsuki, Tanida, Matsuda. Approved the final version of the manuscript on behalf of all authors: Ito. Statistical analysis: Tanida. Administrative/technical/material support: Tanida, Okamoto, Matsuda. Study supervision: Fujibayashi, Tanida, Okamoto, Matsuda.

\section{Correspondence}

Takayuki Ito: Japanese Red Cross Wakayama Medical Center, Wakayama City, Wakayama, Japan. itotak0119@gmail.com. 